\title{
COMPARISON RESULTS FOR CAPACITY
}

\author{
ANA HURTADO*, VICENTE PALMER\#, AND MANUEL RITORÉ*
}

\begin{abstract}
We obtain in this paper bounds for the capacity of a compact set $K$. If $K$ is contained in an $(n+1)$-dimensional Cartan-Hadamard manifold, has smooth boundary, and the principal curvatures of $\partial K$ are larger than or equal to $H_{0}>0$, then $\operatorname{Cap}(K) \geqslant(n-1) H_{0} \operatorname{vol}(\partial K)$. When $K$ is contained in an $(n+1)$-dimensional manifold with non-negative Ricci curvature, has smooth boundary, and the mean curvature of $\partial K$ is smaller than or equal to $H_{0}$, we prove the inequality $\operatorname{Cap}(K) \leqslant(n-1) H_{0} \operatorname{vol}(\partial K)$. In both cases we are able to characterize the equality case. Finally, if $K$ is a convex set in Euclidean space $\mathbb{R}^{n+1}$ which admits a supporting sphere of radius $H_{0}^{-1}$ at any boundary point, then we prove $\operatorname{Cap}(K) \geqslant(n-1) H_{0} \mathcal{H}^{n}(\partial K)$ and that equality holds for the round sphere of radius $H_{0}^{-1}$.
\end{abstract}

\section{INTRODUCTION}

The capacity of a compact set $K$ in a Riemannian manifold $M$ is defined by

$$
\operatorname{Cap}(K)=\inf \left\{\int_{M}|\nabla \phi|^{2} d V: \phi \in \mathcal{L}(K)\right\},
$$

where $\mathcal{L}(K)$ is the set of functions in the Sobolev space $H_{0}^{1}(M)$ with $0 \leqslant \phi \leqslant 1$ and $\left.\phi\right|_{K} \equiv 1$, and $d V$ is the Riemannian volume in $M$.

From a physical point of view, the capacity of a compact set $K$ represents the total electric charge flowing into $M \backslash K$ through the boundary $\partial K$. The exact value of the capacity of a set is known in few cases, and so its estimation in geometrical terms is of great interest, not only in electrostatic, but in many physical descriptions of flows, fluids, or heat, where Laplace equations is used, 5 . On the other hand, estimates of the capacity are enough to get geometrical consequences such as the parabolic or hyperbolic character of the manifold, [14, [13, [17, [18. We refer to the survey by A. Grigor'yan [10] for a discussion of these and related concepts.

The employment of geometrical and comparison techniques allows to obtain geometric inequalities involving capacity, 24, 223, 20, p. 17]. A bound for the capacity of a compact set with analytic boundary $K \subset \mathbb{R}^{3}$ was obtained by Szëgo [24, $\S 2$ (15)] in terms of the integral of the mean curvature $H$. He proved

$$
\operatorname{Cap}(K) \leqslant \int_{\partial K} H d A
$$

with equality for the round ball.

Date: March 27, 2013.

2010 Mathematics Subject Classification. 31C12, 31C15, 53C21, 58J65, 35J25.

Key words and phrases. capacity, equilibrium potential, hyperbolicity, mean curvature, CartanHadamard manifolds.

* Supported by MICINN-FEDER grant MTM2010-21206-C02-01, and Junta de Andalucía grants FQM-325 and P09-FQM-5088.

\# Supported by the Caixa Castelló Foundation, and DGI grant MTM2010-21206-C02-02. 
Szëgo also showed that, for a compact set $K \subset \mathbb{R}^{3}$ with analytic boundary, one has

$$
\operatorname{Cap}(K) \geqslant\left(\frac{3}{4 \pi}\right)^{1 / 3} \operatorname{vol}(K)^{1 / 3},
$$

with equality precisely for the round ball, [23, $\S 2$, solving a problem stated by Poincaré in 1903 with an incomplete variational proof [20, p. vi]. In fact, Szëgo's proof works for compact sets $K \subset \mathbb{R}^{n+1}, n \geqslant 2$, with smooth boundary, and provides the inequality

$$
\operatorname{Cap}(K) \geqslant c_{n+1}^{2} \frac{n-1}{n+1} \operatorname{vol}(K)^{(n-1) /(n+1)},
$$

with equality for the round ball. Here $c_{n+1}$ is the isoperimetric constant that appears in the optimal isoperimetric inequality $\operatorname{vol}(\partial K) \geqslant c_{n+1} \operatorname{vol}(K)^{n /(n+1)}$ in $\mathbb{R}^{n+1}$. Inequality (1.2) holds for any compact set $K \subset \mathbb{R}^{n+1}$ by [10, Thm. 8.1].

Polya and Szëgo also conjectured that there is a positive constant $\kappa$ such that

$$
\frac{\operatorname{Cap}(K)}{\operatorname{vol}(\partial K)^{1 / 2}} \geqslant \kappa,
$$

for any convex set $K \subset \mathbb{R}^{3}$ (the above quantity has no lower bound for sets with nonconvex boundary). They also conjectured that $\kappa=(32)^{1 / 2} \pi^{-1 / 2}$ and that equality holds for planar disks, see [5], 20, 25].

In this paper we prove two more results on estimations of the capacity of a compact set. In Theorem 3.1 we obtain

Let $M^{n+1}$ be a Cartan-Hadamard manifold, and $K \subset M^{n+1}$ a compact set with smooth boundary so that the principal curvatures of $\partial K$ are larger than or equal to some constant $H_{0}>0$. Then we have

$$
\operatorname{Cap}(K) \geqslant(n-1) H_{0} \operatorname{vol}(\partial K) .
$$

Moreover, we are able to characterize the equality: it is attained if and only if the convex body has umbilical boundary and $M \backslash \operatorname{int}(K)$ is isometric to a warped product. This result is optimal, as will be shown by Remark 3.2. As a consequence of this theorem, we obtain a simple proof of the well-known fact that a Cartan-Hadamard manifold of dimension at least three is hyperbolic.

On the other hand, for manifolds with non-negative Ricci curvature, we prove in Theorem 3.5

Let $M^{n+1}$ be a complete non-compact Riemannian manifold with nonnegative Ricci curvature, and $K \subset M^{n+1}$ a compact set with smooth boundary. Assume that the mean curvature of $\partial K$ is smaller than or equal to $H_{0}>0$. Then

$$
\operatorname{Cap}(K) \leqslant(n-1) H_{0} \operatorname{vol}(\partial K) .
$$

We are also able to characterize the equality case. It is worthy to point out that for analytic compact sets in $\mathbb{R}^{3}$, the above result can be also obtained by applying the estimation (1.1) in terms of the mean curvature given by Szëgo [24].

In the proof of both results we use a transposition of the equilibrium potential of the Euclidean ball of radius $H_{0}^{-1}$ to the exterior of $K$ by means of the distance function to $K$. In the Cartan-Hadamard case there are no problems of differentiability, since the distance to a convex set with $C^{\infty}$ boundary is a $C^{\infty}$ function. In the non-negative Ricci curvature case, although the distance to $\partial K$ is only smooth out of the cut locus of $\partial K$, the technical difficulties introduced by the cut locus can be handled by a method of Cheeger and Yau [4. Theorems 3.1 and 3.5 were proven by Ichihara [13, [14] for geodesic balls, see also [10, § 15]. 
Theorems 3.1 and 3.5 are valid in the Euclidean space $\mathbb{R}^{n+1}$. In both results equality holds when $K$ is a Euclidean ball. Assuming $K$ is convex, Minkowski's formula implies the inequality $\operatorname{vol}(\partial K) \geqslant(n+1) H_{0} \operatorname{vol}(K)$ when the principal curvatures of $\partial K$ are larger than or equal to $H_{0}>0$, and it implies the opposite inequality when $H \leqslant H_{0}$. We conclude in Corollary 4.3 that

$$
\operatorname{Cap}(K) \geqslant\left(n^{2}-1\right) H_{0}^{2} \operatorname{vol}(K),
$$

when the principal curvatures of $\partial K$ are larger than or equal to $H_{0}$, and we conclude the opposite inequality in Corollary 4.4 when the mean curvature of $\partial K$ is smaller than or equal to $H_{0}$. In both Corollaries, equality holds for the round sphere.

In Euclidean space we can define a weak notion of "principal curvatures bounded from below". A compact set $K$ in Euclidean space is $\lambda$-convex, $\lambda>0$, if there is a supporting ball of radius $\lambda^{-1}$ at every boundary point of $K$. A set $K$ with smooth boundary is $\lambda$-convex if and only if the principal curvatures of the boundary are larger than or equal to $\lambda$ [21, p. 502], and so $\lambda$-convexity is an extension of the inequalities $\kappa_{i} \geqslant \lambda$, where $\kappa_{i}$ are principal curvatures, in a weak sense. We are able to extend the estimate in Theorem 3.1 to $H_{0}$-convex sets in Euclidean space without assuming the smoothness of the boundary. We prove in Theorem 4.5

$$
\begin{aligned}
& \text { Let } K \text { be an } H_{0} \text {-convex body in } \mathbb{R}^{n+1}, H_{0}>0 \text {. Then } \\
& \qquad \operatorname{Cap}(K) \geqslant(n-1) H_{0} \mathcal{H}^{n}(\partial K), \\
& \text { where } \mathcal{H}^{n} \text { is the } n \text {-dimensional Hausdorff measure. Equality holds if } \\
& \text { and only if } K \text { is a round ball of radius } H_{0}^{-1} \text {. }
\end{aligned}
$$

We have organized this paper into three sections apart from this introduction. Section 2 is devoted to establish the definitions and results related to the capacity of a compact set, which we will need in the sequel. In Section 3, we formulate and prove our main results, Theorems 3.1 and 3.5 for the capacity of compact sets in manifolds with a suitable control of their curvatures. Finally, in Section 4, we state some consequences of Theorems 3.1 and 3.5 in the Euclidean space and we prove Theorem 4.5 .

\section{Preliminaries}

Given an open set $\Omega$ in a Riemannian manifold $M$ and a compact set $K \subset \Omega$, we define the capacity of $K$ in $\Omega$ as

$$
\operatorname{Cap}(K, \Omega)=\inf \left\{\int_{\Omega}|\nabla \phi|^{2} d V: \phi \in \mathcal{L}(K, \Omega)\right\},
$$

where $\mathcal{L}(K, \Omega)$ is the set of functions on $M$ with compact support in $\bar{\Omega}$ which are locally Lipschitz and satisfy: $0 \leqslant \phi \leqslant 1$ and $\phi_{\mid K}=1$, see [10]. Here $d V$ is the Riemannian volume of $M$.

When $\Omega$ is a precompact set and both $\Omega$ and $K$ have smooth boundary, the infimum in (2.1) is attained by the unique solution of the Dirichlet problem in $\Omega \backslash K$

$$
\left\{\begin{aligned}
\Delta u=0 & \text { on } \Omega \backslash K, \\
u=1 & \text { on } \partial K, \\
u=0 & \text { on } \partial \Omega .
\end{aligned}\right.
$$

The function $u$ is called the equilibrium potential of $(K, \Omega)$. Using Green's formulae, we obtain

$$
\operatorname{Cap}(K, \Omega)=\int_{\Omega \backslash K}|\nabla u|^{2} d V=\int_{\partial K}\langle\nabla u, \nu\rangle d A=\int_{\partial K}|\nabla u| d A,
$$


where $\nu$ is the unit normal vector field along $\partial K$ pointing into $K$, and $d A$ is the Riemannian area element of $\partial K$.

The capacity can be defined in the whole manifold $M$ by considering any exhaustion sequence $\left\{\Omega_{n}\right\}_{n \in \mathbb{N}}$ covering $M$ such that $K \subset \Omega_{n} \subset \bar{\Omega}_{n} \subset \Omega_{n+1}$ for all $n \in \mathbb{N}$. Then,

$$
\operatorname{Cap}(K)=\lim _{n \rightarrow \infty} \operatorname{Cap}\left(K, \Omega_{n}\right) \text {. }
$$

Moreover, if $\left\{\Omega_{n}\right\}_{n \in \mathbb{N}}$ is an exhaustion by precompact sets such that $K \subset \Omega_{n}$ for all $n \in \mathbb{N}$, the maximum principle for elliptic operators implies that $u_{n+1} \geqslant u_{n}$ in $\Omega_{n} \backslash K$. Hence the limit

$$
u=\lim _{n \rightarrow \infty} u_{n}
$$

exists and is a harmonic function with $u=1$ on $\partial K$. A second application of the maximum principle implies that $u$ is independent of the exhaustion by precompact sets considered. It follows easily that

$$
\operatorname{Cap}(K)=\inf \left\{\int_{M}|\nabla \phi|^{2} d V: \phi \in \mathcal{L}(K)\right\},
$$

where $\mathcal{L}(K)$ is the set of functions in the Sobolev space $H_{0}^{1}(M)$ with $0 \leqslant \phi \leqslant 1$ and $\left.\phi\right|_{K} \equiv 1$. The function $u$ is called the equilibrium potential of $K$ and satisfies

$$
\operatorname{Cap}(K)=\int_{\partial K}|\nabla u| d A .
$$

For compact sets with non-smooth boundary, the equilibrium potential $u$ can be obtained as the unique limit of the equilibrium potentials $u_{n}$ of a nested sequence $\left\{K_{n}\right\}$ of approximating smoothly bounded compact sets. Then $u$ is a harmonic $C^{\infty}$ function in $M \backslash K$.

If $K$ is a convex body in Euclidean space, the equilibrium potential $u$ extends continuously $\mathcal{H}^{n}$-almost everywhere to $\partial K[\underline{6}$, and the gradient of $u$ has a non-tangential limit to $\partial K$ except in a set of $\mathcal{H}^{n}$-measure zero [15]. Also from [15] it follows that formula (2.3) holds in the non-smooth case.

For comparison purposes we are going to compute explicitly the capacity and the equilibrium potential of the $(n+1)$-dimensional closed ball $\bar{B}_{1 / H_{0}} \subset \mathbb{R}^{n+1}$ of mean curvature $H_{0}>0$.

By symmetry, we easily see that the equilibrium potential of $\left(\bar{B}_{1 / H_{0}}, B_{\left(1 / H_{0}\right)+t}\right)$ only depends on the distance function $r$ to $\partial \bar{B}_{1 / H_{0}}$ and is given, for $n \geqslant 2$, by

$$
\Phi_{t}(r)=\frac{1}{1-\left(1+t H_{0}\right)^{1-n}}\left(\left(1+r H_{0}\right)^{1-n}-\left(1+t H_{0}\right)^{1-n}\right) .
$$

In fact,

$$
\Delta \Phi_{t}=\Phi_{t}^{\prime \prime}(r)+\frac{n H_{0}}{1+r H_{0}} \Phi_{t}^{\prime}(r)=0,
$$

and the boundary conditions $\Phi_{t}(0)=1$ and $\Phi_{t}(t)=0$ are satisfied. So, $\Phi_{t}$ is a solution of the corresponding Dirichlet problem in $B_{\left(1 / H_{0}\right)+t} \backslash \bar{B}_{1 / H_{0}}$ given by (2.2). Moreover, the maximum principle for elliptic operators guarantees that $\Phi_{t}$ is the only solution.

Taking limits in (2.4) when $t \rightarrow \infty$ we obtain that the equilibrium potential of $\bar{B}_{1 / H_{0}}$ is given by

$$
\Phi_{H_{0}}(r)=\left(1+r H_{0}\right)^{1-n} .
$$

As a consequence of (2.3) and equality $\left|\nabla \Phi_{H_{0}}\right|=-\Phi_{H_{0}}^{\prime}(0)=(n-1) H_{0}$ in $\partial \bar{B}_{1 / H_{0}}$ we get

$$
\operatorname{Cap}\left(\bar{B}_{1 / H_{0}}\right)=(n-1) H_{0} \operatorname{vol}\left(\partial \bar{B}_{1 / H_{0}}\right) .
$$


Formula (2.7) also holds for $n=1$, since in this case $\Phi_{t}(r)=1-\log \left(1+H_{0} r\right) / \log (1+$ $\left.H_{0} t\right), \Phi(r) \equiv 1$, and $\operatorname{Cap}\left(\bar{B}_{1 / H_{0}}\right)=0$.

\section{MAin RESUlts}

We begin this section with a comparison result for the capacity of a convex body in a Cartan-Hadamard manifold. In the sequel, by a convex body we mean a compact convex body set with nonempty interior. By a result of Alexander [1, Thm. 1], a compact set with smooth boundary and positive principal curvatures in a CartanHadamard manifold is a convex set.

Theorem 3.1. Let $M^{n+1}$ be a Cartan-Hadamard manifold, and $K \subset M^{n+1}$ a compact set with smooth boundary. Assume that the principal curvatures of $\partial K$ are larger than or equal to some constant $H_{0}>0$. Then we have

$$
\operatorname{Cap}(K) \geqslant(n-1) H_{0} \operatorname{vol}(\partial K) \text {. }
$$

Equality holds in (3.1) if and only if $\partial K$ is totally umbilical with mean curvature $H_{0}$ and $M \backslash \operatorname{int}(K)$ is isometric to the product $\partial K \times[0, \infty)$ with the warped metric $(1+$ $\left.H_{0} r\right)^{2} h+d r^{2}$, where $h$ is the Riemannian metric in $\partial K$ and $r \in[0, \infty)$.

Proof. Consider the equilibrium potential $\Phi=\Phi_{H_{0}}$ of the Euclidean ball $\bar{B}_{1 / H_{0}}$. Let $r: M \backslash \operatorname{int}(K) \rightarrow \mathbb{R}$ denote the distance to $K$ [2]. The function $r$ is $C^{\infty}$ in $M \backslash K$ since the cut locus of a convex set in a Cartan-Hadamard manifold is empty. We define $v: M \backslash \operatorname{int}(K) \rightarrow \mathbb{R}$ by

$$
v(p)=\Phi_{H_{0}}(r(p)) .
$$

It is clear that $0 \leqslant v \leqslant 1$, that $v \equiv 1$ in $\partial K$, and that function $v$ is $C^{\infty}$ in $M \backslash K$. We have

$$
\Delta v(p)=\Phi^{\prime \prime}(r(p))+\Phi^{\prime}(r(p)) n H_{r}(p)
$$

where $H_{r}$ is the mean curvature of $\partial K_{r}$.

We estimate the mean curvature $H_{r}$ as in Rauch's Comparison Theorem [3, II.6.4]. Let $\gamma:[0, \infty) \rightarrow M$ be a geodesic leaving $\partial K$ orthogonally and $E$ a Jacobi field along $\gamma$ orthogonal to $\gamma^{\prime}(r)$ for all $r \geqslant 0$. Define

$$
f(r)=\frac{\left\langle E^{\prime}, E\right\rangle(r)}{|E(r)|^{2}}
$$

where $E^{\prime}$ is the covariant derivative of $E$ along $\gamma$. Computing the derivative of $f(r)$ using the Jacobi equation we have

$$
f^{\prime}(r)=-\frac{R\left(E, \gamma^{\prime}, \gamma^{\prime}, E\right)}{|E|^{2}}(r)+\frac{\left|E^{\prime}\right|^{2}|E|^{2}-\left\langle E^{\prime}, E\right\rangle^{2}}{|E|^{4}}(r)-f(r)^{2} \geqslant-f(r)^{2},
$$

where in the inequality we have used that the sectional curvature $R\left(E, \gamma^{\prime}, \gamma^{\prime}, E\right)$ of the plane generated by $E(r)$ and $\gamma^{\prime}(r)$ is non-negative, and that $\left\langle E^{\prime}, E\right\rangle^{2} \leqslant|E|^{2}\left|E^{\prime}\right|^{2}$ by Cauchy-Schwarz inequality. Integrating the above differential inequality, we obtain

$$
f(r) \geqslant \frac{f(0)}{1+f(0) r} \text {. }
$$

Equality holds in (3.5) if and only if the sectional curvature along the plane generated by $\gamma^{\prime}(r)$ and $E(r)$ is 0 for all $r \geqslant 0$ and $E /|E|$ is a parallel vector field along $\gamma$ (observe that $\left(|E|^{-1} E\right)^{\prime}$ has modulus $|E|^{-2}\left(|E|^{2}\left|E^{\prime}\right|^{2}-\left\langle E, E^{\prime}\right\rangle^{2}\right)^{1 / 2}$.

Fix now some $r>0$. To estimate the mean curvature $H_{r}(p)$ we choose Jacobi fields $E_{1}, \ldots, E_{n}$ along $\gamma$ which are independent eigenvectors of the second fundamental 
form of $\partial K_{r}$ at $p$. Hence they are everywhere orthogonal to $\gamma^{\prime}$. For $i=1, \ldots, n$ we consider the functions along $\gamma$ defined by

So we have

$$
f_{i}=\frac{\left\langle E_{i}^{\prime}, E_{i}\right\rangle}{\left|E_{i}\right|^{2}}
$$

$$
n H_{r}(p)=\sum_{i=1}^{n} \frac{\left\langle E_{i}^{\prime}, E_{i}\right\rangle}{\left|E_{i}\right|^{2}}(r(p)) \geqslant \sum_{i=1}^{n} \frac{f_{i}(0)}{1+f_{i}(0) r(p)} \geqslant \frac{n H_{0}}{1+H_{0} r(p)} .
$$

The first inequality in (3.6) follows from (3.5) and the second one since $f_{i}(0) \geqslant H_{0}$ and the function $x \mapsto x /(1+r x)$ is increasing. Equality holds in (3.6) for all $i=1, \ldots, n$ if and only if the principal curvatures of $\partial K$ at $\gamma(0)$ are all equal to $H_{0}$, the sectional curvatures of the planes generated by $\gamma^{\prime}(r)$ and $E_{i}(r)$ are identically 0 , and $E_{i} /\left|E_{i}\right|$ is a parallel vector field. Assuming $\left|E_{i}(0)\right|=1$ for all $i=1, \ldots, n$, we get

$$
E_{i}(r)=\left(1+H_{0} r\right) P_{i}(r),
$$

where $P_{i}(r)$ is a parallel vector field along $\gamma$. This follows since $\left|E_{i}(r)\right|=1+H_{0} r$, because $\left(\left|E_{i}\right|^{2}\right)^{\prime}=2\left\langle E_{i}^{\prime}, E_{i}\right\rangle=2 f\left|E_{i}\right|^{2}$ and $f(r)=H_{0}\left(1+H_{0} r\right)^{-1}$ as we are assuming that equality holds in (3.6). Assume that (3.7) holds for every point in $\partial K$. Consider the map $f: \partial K \times[0, \infty) \rightarrow M \backslash \operatorname{int}(K)$ given by $f(p, r):=\exp _{p}\left(r N_{p}\right)$, where $N_{p}$ is the outer unit normal to $\partial K$. The pullback of the Riemannian metric of $M \backslash \operatorname{int}(K)$ can be written on $\partial K \times[0, \infty)$ as the warped metric

$$
\left(1+H_{0} r\right)^{2} h+d r^{2}
$$

where $h$ is the Riemannian metric of $\partial K$.

Let $u$ be the equilibrium potential of $K$. Since $\Phi^{\prime} \leqslant 0$, we conclude from (2.5), (3.2) and (3.6) that

$$
\Delta v(p) \leqslant \Phi^{\prime \prime}(r(p))+\Phi^{\prime}(r(p)) \frac{n H_{0}}{1+H_{0} r(p)}=0=\Delta u(p) .
$$

Let us check that $v \geqslant u$ in $M \backslash K$. For every $t>0$, let $u_{t}$ be the equilibrium potential of $\left(K, K_{t}\right), \Phi_{t}$ the equilibrium potential in Euclidean space of $\left(\bar{B}_{1 / H_{0}}, B_{\left(1 / H_{0}\right)+t}\right)$, and $v_{t}:=\Phi_{t} \circ r$. Equation (3.6) implies that $\Delta\left(v_{t}-u_{t}\right) \leqslant 0$ in $K_{t} \backslash K$. By the maximum principle, $v_{t} \geqslant u_{t}$ in $K_{t} \backslash K$. Since $v_{t}, u_{t}$ are increasing families converging to $v$ and $u$ respectively we conclude that $v \geqslant u$ in $M \backslash K$.

As $\Delta(v-u) \leqslant 0$ in $M \backslash K, v \geqslant u$ in $M \backslash K$, and $u \equiv v \equiv 1$ in $\partial K$, the maximum principle [9, § 3.2] implies

$$
|\nabla v| \leqslant|\nabla u| \quad \text { on } \partial K
$$

and so

$$
\begin{aligned}
\operatorname{Cap}(K)=\int_{\partial K}|\nabla u| d A & \geqslant \int_{\partial K}|\nabla v| d A \\
& =-\Phi^{\prime}(0) \operatorname{vol}(\partial K)=(n-1) H_{0} \operatorname{vol}(\partial K) .
\end{aligned}
$$

Assume equality holds in (3.9). Then $|\nabla v|=|\nabla u|$ on $\partial K$ and, by the strong maximum principle [9], $u \equiv v$ on $M \backslash K$. This implies that equality also holds in (3.8) and (3.6). By the discussion of equality after (3.6) we have that the principal curvatures of $\partial K$ are all equal to $H_{0}$ and $M \backslash \operatorname{int}(K)$ is isometric to the product $\partial K \times[0, \infty)$, with the warped metric $\left(1+H_{0} r\right)^{2} h+d r^{2}$, where $h$ is the Riemannian metric in $\partial K$ and $r \in[0, \infty)$.

Remark 3.2. The characterization of equality in Theorem 3.1 is the best possible. Consider a smooth function $g:[0, \infty) \rightarrow[0, \infty)$ so that $g(0)=0, g^{\prime}(0)=1, g^{(2 k)}(0)=$ 0 for all $k \in \mathbb{N}, g^{\prime \prime}(t) \geqslant 0$ for all $t \geqslant 0$. Then $\mathbb{S}^{n} \times[0, \infty)$ with the warped metric 
$g(t)^{2} h_{0}+d t^{2}$, where $h_{0}$ is the standard Riemannian metric in $\mathbb{S}^{n}$, is a smooth complete $(n+1)$-dimensional Hadamard manifold $M$ [19, $\S 3.2 .3]$. Given $t_{0}>0, H_{0}>0$, the function $g$ can be chosen so that we have $H_{0}=g^{\prime}\left(t_{0}\right) / g\left(t_{0}\right)$ and

$$
g\left(t_{0}+r\right)=g\left(t_{0}\right)\left(1+H_{0} r\right), \quad r \geqslant 0 .
$$

Consider the convex ball $K:=\left\{t \leqslant t_{0}\right\}$. The principal curvatures of $\partial K$ are all equal to $H_{0}$, and the mean curvature of $\partial K_{r}=\left\{t=t_{0}+r\right\}$ is given by

$$
H_{r}=\frac{g^{\prime}\left(t_{0}+r\right)}{g\left(t_{0}+r\right)}=\frac{H_{0}}{1+H_{0} r},
$$

so that the equilibrium potential of $K$ is given by $u(r)=\Phi_{H_{0}}(r)$, where $\Phi_{H_{0}}$ is defined by (2.6). Then we have

$$
\operatorname{Cap}(K)=\int_{\partial K}|\nabla u| d A=-\Phi_{H_{0}}^{\prime}(0) \operatorname{vol} \partial K=(n-1) H_{0} \operatorname{vol}(\partial K) .
$$

In this example, the Riemannian metric inside $K$ can be slightly perturbed (around a point with strictly negative sectional curvatures) to a non-warped metric with nonpositive sectional curvatures.

Remark 3.3. The inequality of Theorem 3.1, can be also written as

$$
\frac{\operatorname{Cap}(K)}{\operatorname{vol}(\partial K)} \geqslant \frac{\operatorname{Cap}\left(\bar{B}_{1 / H_{0}}\right)}{\operatorname{vol}\left(\partial \bar{B}_{1 / H_{0}}\right)}
$$

using (2.7) whenever $K \subset M$ is a compact set with smooth boundary and principal curvatures satisfying $\kappa_{i} \geqslant H_{0}$. So we have obtained a comparison result between the capacity of a convex body in $M$ and the capacity of a round ball in the Euclidean space via the previous comparison of the Laplacian of the distance function in both manifolds.

Remark 3.4. A Riemannian manifold $M$ is said to be hyperbolic if there exists a nonconstant positive superharmonic function on $M$. Otherwise it is called parabolic. The so-called type problem for manifolds is related to the problem of establishing necessary and sufficient geometric conditions for a Riemannian manifold to be hyperbolic or parabolic. This classical problem began to be studied for Riemannian surfaces in the thirties by Ahlfors, Myrberg, Nevanlinna and Royden among others and have given rise to a large literature.

Lyons and Sullivan [16] gave a list of equivalent conditions to check the hyperbolicity of an oriented Riemannian manifold, which is known as the Kelvin-NevanlinnaRoyden criterium. This criterium states that $M$ is hyperbolic if and only if there exists a compact set $K$ in $M$ with positive capacity.

Notice that, as a consequence of the above theorem, the capacity of a geodesic ball of a Cartan-Hadamard manifold of dimension greater than or equal to three is strictly positive. So, applying the Kelvin-Nevanlinna-Royden criterium we have an alternative proof of the hyperbolicity of such manifolds. This was previously known by the works of Ichihara [13, 14, see also [10, Thm. 15.3]. Alternatively, one can also use the isoperimetric inequality of Hoffman and Spruck [12] together with Theorem 8.2 in [10] to prove the hyperbolicity of Cartan-Hadamard manifolds of dimension larger than or equal to three.

Now we state a comparison result for complete non-compact manifolds with nonnegative Ricci curvature. 
Theorem 3.5. Let $M^{n+1}$ be a complete non-compact Riemannian manifold with non-negative Ricci curvature, and $K \subset M^{n+1}$ a compact set with smooth boundary. Assume that the mean curvature of $\partial K$ is smaller than or equal to $H_{0}>0$. Then

$$
\operatorname{Cap}(K) \leqslant(n-1) H_{0} \operatorname{vol}(\partial K) .
$$

Moreover, equality holds in (3.10) if and only if $M \backslash \operatorname{int}(K)$ is isometric to the product $\partial K \times[0, \infty)$ with the warped metric $\left(1+H_{0} r\right)^{2} h+d r^{2}$, where $h$ is the Riemannian metric of $\partial K$, and $r \in[0, \infty)$.

Proof. As in the proof of Theorem 3.1. we consider the function $v(p)=\Phi_{H_{0}}(r(p))$, which is Lipschitz in $M \backslash K$ and smooth on $M \backslash\left(K \cup C_{\partial K}\right)$, where $C_{\partial K}$ is the cut locus of $\partial K$ in $M \backslash K$. Recall that $C_{\partial K}$ is a closed set of Riemannian measure zero on $M \backslash K$. Moreover, if $p \in M \backslash\left(K \cup C_{\partial K}\right)$, then there is a unique minimizing geodesic connecting $p$ and $\partial K$, which is entirely contained in $M \backslash\left(K \cup C_{\partial K}\right)$. We have

$$
\Delta v(p)=\Phi^{\prime \prime}(r(p))+\Phi^{\prime}(r(p)) n H_{r}(p) .
$$

Consider a geodesic $\gamma:[0, c(p)) \rightarrow M$ minimizing the distance to $\partial K$ with $\gamma(0)=p$, where $c(p)$ is the cut distance. The derivative of the mean curvature $H_{r}$ of the parallel hypersurface $\partial K_{r}$ along $\gamma$ is given by

$$
n H_{r}^{\prime}=-\operatorname{Ric}\left(\gamma^{\prime}, \gamma^{\prime}\right)-\left|\sigma_{r}\right|^{2} \leqslant-\left|\sigma_{r}\right|^{2} \leqslant-n H_{r}^{2}
$$

where $\left|\sigma_{r}\right|^{2}$ is the squared norm of the second fundamental form of $\partial K_{r}$, i.e., the sum of the squared principal curvatures. Equality holds in (3.12) if and only if $\operatorname{Ric}\left(\gamma^{\prime}, \gamma^{\prime}\right)=0$ and $\left|\sigma_{r}\right|^{2}=n H_{r}^{2}$ (when $\partial K_{r}$ is totally umbilical at $\gamma(r)$ ). Hence, from the differential inequality (3.12), we conclude

$$
H_{r}(\gamma(r)) \leqslant \frac{H_{0}(p)}{1+H_{0}(p) r} \leqslant \frac{H_{0}}{1+H_{0} r} .
$$

In case of equality in (3.13), we have $\operatorname{Ric}\left(\gamma^{\prime}, \gamma^{\prime}\right) \equiv 0$ along $\gamma,\left|\sigma_{r}\right|^{2}=n H_{r}^{2}$, and $H_{r}=H_{0}\left(1+H_{0} r\right)^{-1}$. In particular, $\partial K_{r}$ is totally umbilical with principal curvatures $H_{0}\left(1+H_{0} r\right)^{-1}$. Hence, if $E$ is a Jacobi field along the geodesic $\gamma$ orthogonal to $\gamma$ we have

$$
E^{\prime}(r)=\frac{H_{0}}{1+H_{0} r} E(r)
$$

It is easy to get from this equation that $E^{\prime \prime}=0$. By the Jacobi equation the sectional curvature $R\left(\gamma^{\prime}, E, E, \gamma^{\prime}\right)$ of the plane generated by $E$ and $\gamma^{\prime}$ is equal to 0 . Formula (3.14) implies that $E$ and $E^{\prime}$ are linearly dependent. So we get $\left\langle E, E^{\prime}\right\rangle^{2}=|E|^{2}\left|E^{\prime}\right|^{2}$ and we conclude, as in the proof of Theorem 3.1 , that $E /|E|$ is a parallel vector field along $\gamma$ and that $|E(r)|=1+H_{0} r$. So it follows, as in the Cartan-Hadamard case, that $M \backslash \operatorname{int}(K)$ is isometric to the product $\partial K \times[0, \infty)$ with the warped metric $\left(1+H_{0} r\right)^{2} h+d r^{2}$, where $h$ is the Riemannian metric of $\partial K$.

From (3.11) and (3.13) we get

$$
\Delta v(p) \geqslant 0, \quad \text { in } \quad M \backslash\left(K \cup C_{\partial K}\right) .
$$

We can prove that the above inequality is also true in $M \backslash K$ in the sense of distributions. Indeed, let us show, following the method of Cheeger and Yau 44 (see also [10]), that for all non-negative $\phi \in C_{0}^{\infty}(M \backslash K)$ we have that

$$
\langle\Delta v, \phi\rangle:=-\int_{M \backslash K}\langle\nabla v, \nabla \phi\rangle d V \geqslant 0 .
$$

Given $p \in \partial K$, let $c(p)$ be the cut distance (possibly $\infty$ ). Since the cut locus $C_{\partial K}$ is a closed set, the function $c(p)$ is lower semi-continuous, so it can be obtained as 
the limit of an increasing sequence $\left\{c_{k}(p)\right\}$ of smooth positive functions. Now, let us define

$$
V_{k}:=\left\{\exp _{p}\left(r N_{p}\right): p \in \partial K, 0<r<c_{k}(p)\right\}
$$

where $N_{p}$ is the unit outer normal to $\partial K$.

In this way, we obtain an increasing sequence $\left\{V_{k}\right\}$ of open sets with smooth boundary such that $\bigcup_{k} V_{k}=M \backslash\left(K \cup C_{\partial K}\right)$. Then, applying Green's formulae

$$
\begin{aligned}
\int_{V_{k}}\langle\nabla v, \nabla \phi\rangle d V & =-\int_{V_{k}} \phi \Delta v d V-\int_{\partial V_{k}} \phi\left\langle\nabla v, \nu_{k}\right\rangle d A \\
& =-\int_{V_{k}} \phi \Delta v d V-\int_{\partial V_{k}} \phi \Phi^{\prime}\left\langle\nabla r, \nu_{k}\right\rangle d A
\end{aligned}
$$

where $\nu_{k}$ is the unit inner normal vector field along $\partial V_{k}$. We have also used $\phi \equiv 0$ in $\partial V_{k} \cap \partial K$. As $V_{k}=\left\{\exp _{p}\left(c_{k}(p) N_{p}\right): p \in \partial K\right\}$ is a radial graph over $\partial K, \nabla r$ is never tangent to $\partial V_{k}$, and hence $\left\langle\nabla r, \nu_{k}\right\rangle \neq 0$. As $\nu_{k}$ is the inner unit normal to $\partial V_{k}$, we have $\left\langle\nabla r, \nu_{k}\right\rangle<0\left(\nu_{k}\right.$ and $\nabla r$ form an obtuse angle). Taking into account (3.15) and $\Phi^{\prime} \leqslant 0$, we conclude

$$
\int_{V_{k}}\langle\nabla v, \nabla \phi\rangle d V \leqslant 0
$$

Since $C_{\partial K}$ has measure zero, taking $k \rightarrow \infty$, we obtain (3.16).

As in the proof of Theorem 3.1 for every $t>0$, let $u_{t}$ be the equilibrium potential of $\left(K, K_{t}\right), \Phi_{t}$ the equilibrium potential in Euclidean space of $\left(\bar{B}_{1 / H_{0}}, B_{\left(1 / H_{0}\right)+t}\right)$, and $v_{t}:=\Phi_{t} \circ r$. By the method exposed above $\Delta\left(v_{t}-u_{t}\right) \geqslant 0$ in $K_{t} \backslash K$ in the sense of distributions and applying the weak maximum principle, [9, $\S 3.1$ ], we get $v_{t} \leqslant u_{t}$ in $K_{t} \backslash K$. Since $v_{t}, u_{t}$ are increasing families converging to $v$ and $u$ respectively we conclude that $v \geqslant u$ in $M \backslash K$.

Hence we can argue as in the proof of Theorem 3.1 to conclude that

$$
|\nabla v| \geqslant|\nabla u| \text { on } \partial K
$$

and so

$$
\operatorname{Cap}(K)=\int_{\partial K}|\nabla u| d A \leqslant \int_{\partial K}|\nabla v| d A=-\Phi^{\prime}(0) \operatorname{vol}(\partial K)=(n-1) H_{0} \operatorname{vol}(\partial K) .
$$

Equality holds when $u=v$ in $M \backslash\left(\partial K \cup C_{\partial K}\right)$. So equality holds in (3.12) and (3.13). By the previous discussion of equality, this implies that $M \backslash \operatorname{int}(K)$ is isometric to the product $\partial K \times[0, \infty)$ with the warped metric $\left(1+H_{0} r\right)^{2} h+d r^{2}$, where $h$ is the Riemannian metric of $\partial K$.

Remark 3.6. Note that the inequality obtained for the capacity of $K$, can be also written as

using (2.7).

$$
\frac{\operatorname{Cap}(K)}{\operatorname{vol}(\partial K)} \leqslant \frac{\operatorname{Cap}\left(\bar{B}_{1 / H_{0}}\right)}{\operatorname{vol}\left(\partial \bar{B}_{1 / H_{0}}\right)}
$$

\section{The Euclidean Case}

Hadamard's Theorem [11] implies that a compact set with smooth boundary and strictly positive principal curvatures is a convex set. As a consequence of Theorems 3.1 and 3.5 we get

Corollary 4.1. Let $K \subset \mathbb{R}^{n+1}$ be a convex body with smooth boundary. Assume that the principal curvatures of $\partial K$ are larger than or equal to some constant $H_{0}>0$. Then we have

$$
\operatorname{Cap}(K) \geqslant(n-1) H_{0} \operatorname{vol}(\partial K)
$$


Equality holds in (4.1) if and only if $K$ is a round ball of radius $H_{0}^{-1}$.

Corollary 4.2. Let $K \subset \mathbb{R}^{n+1}$ be a compact set with smooth boundary. Assume that the mean curvature of $\partial K$ is smaller than or equal to some constant $H_{0}>0$. Then we have

$$
\operatorname{Cap}(K) \leqslant(n-1) H_{0} \operatorname{vol}(\partial K)
$$

with equality if and only if $K$ is a round ball of radius $H_{0}^{-1}$.

When $K \subset \mathbb{R}^{n+1}$ is convex, Minkowski formula can be used to relate the capacity of $K$ to its volume. To show this, pick a point $p$ in the interior of $K$, and consider the radial vector field $X$ with center $p$. Let $N$ be the unit inner normal to $\partial K$. By the convexity of $K$ we have $\langle X, N\rangle<0$ in $\partial K$. Let $X^{\top}$ be the tangent projection of the vector field $X$ to $\partial K$. Minkowski formula

$$
\int_{\partial K}(1+H\langle X, N\rangle) d A=0
$$

is obtained by applying the divergence theorem to the vector field $X^{\top}$ in $\partial K$. Under our assumptions we have either $H \leqslant H_{0}$ or $H \geqslant H_{0}$, so that Minkowski formula (4.3) implies

$$
\operatorname{vol}(\partial K)=-\int_{\partial K} H\langle X, N\rangle d A \geqslant H_{0}\left(-\int_{\partial K}\langle X, N\rangle d A\right)
$$

in case $H \geqslant H_{0}$ or the opposite inequality in case $H \leqslant H_{0}$. Since $\operatorname{div} X=(n+1)$ in $\mathbb{R}^{n+1}$, by the divergence theorem applied to $X$ in $K$ we have

$$
\operatorname{vol}(\partial K) \geqslant(n+1) H_{0} \operatorname{vol}(K)
$$

in case $H \geqslant H_{0}$ and the opposite inequality when $H \leqslant H_{0}$.

So we conclude from Corollaries 4.1 and 4.2. and from inequality (4.4)

Corollary 4.3. Let $K \subset \mathbb{R}^{n+1}$ be a convex body with smooth boundary. Assume that the principal curvatures of $\partial K$ are larger than or equal to some constant $H_{0}>0$. Then we have

$$
\operatorname{Cap}(K) \geqslant\left(n^{2}-1\right) H_{0}^{2} \operatorname{vol}(K) .
$$

Equality holds in (4.5) if and only if $K$ is a round ball of radius $H_{0}^{-1}$.

Corollary 4.4. Let $K \subset \mathbb{R}^{n+1}$ be a convex body with smooth boundary. Assume that the mean curvature of $\partial K$ is smaller than or equal to some constant $H_{0}>0$. Then we have

$$
\operatorname{Cap}(K) \leqslant\left(n^{2}-1\right) H_{0}^{2} \operatorname{vol}(K),
$$

with equality if and only if $K$ is a round ball of radius $H_{0}^{-1}$.

Now we consider a general convex body $K \subset \mathbb{R}^{n+1}$, possibly with non-smooth boundary. Given $\lambda>0$, we shall say that $K$ is $\lambda$-convex if, for every $p \in \partial K$, there is a closed ball $B$ of radius $\lambda^{-1}$ such that $K \subset B$ and $p \in \partial B$. Taking outer parallel bodies to both $K$ and $B$ it is immediate to check that the outer parallel body $K_{r}$ is $\left(\lambda^{-1}+r\right)^{-1}$-convex whenever $K$ is $\lambda$-convex. If $K$ has $C^{2}$ boundary then $\lambda$-convexity is equivalent to the lower bound $\kappa_{i} \geqslant \lambda$ for the principal curvatures $\kappa_{i}$ of $\partial K$.

If $K \subset \mathbb{R}^{n+1}$ is a convex body, we will denote by $\xi: \mathbb{R}^{n+1} \backslash \operatorname{int}(K) \rightarrow \partial K$ the metric projection, which is a contractive Lipschitz map [22, § 1.2]. The gradient of the distance function $r: \mathbb{R}^{n+1} \backslash K \rightarrow \mathbb{R}$ is given by

$$
\nabla r(q)=\frac{q-\xi(q)}{r(q)},
$$


and so it is a locally Lipschitz vector field in $\mathbb{R}^{n+1} \backslash K$. Hence $r$ is a $C_{\text {loc }}^{1,1}$ function on $\mathbb{R}^{n+1} \backslash K$, and the gradient of $r$ and $\xi$ are differentiable in the same set. By Rademacher's Theorem, $r$ is twice differenciable $\mathcal{H}^{n+1}$-almost everywhere in $\mathbb{R}^{n+1} \backslash K$. From [4.7), if $\xi$ is differentiable at $q \in \mathbb{R}^{n+1} \backslash K$ then it is also differentiable along the points in the minimizing geodesic connecting $q$ and $K$. So $\nabla r$ is differentiable $\mathcal{H}^{n}$-almost everwhere in the boundary $\partial K_{r}$, for any $r>0$, which implies that $\partial K_{r}$ is a $C^{1,1}$-hypersurface. The second fundamental form and the principal curvatures are defined $\mathcal{H}^{n}$-a.e in $\partial K_{r}$. Moreover, if $K_{r}$ is $\mu$-convex then the principal curvatures $\kappa_{i}$ of $\partial K_{r}$ satisfy $\kappa_{i} \geqslant \mu$.

Principal curvatures can be defined for sets of positive reach [8, [26, $\S 1]$, a class in which convex sets are included. Given $K$, we consider the set of points $p \in \partial K$ where $\xi$ is differentiable in $\xi^{-1}(\{p\})$, which is a set of full $\mathcal{H}^{n}$ measure in $\partial K$ by the discussion above. We intersect this set with the one of regular points, those for which there is a unique supporting hyperplane, which has also full $\mathcal{H}^{n}$ measure 22 Thm. 2.2.4]. Then, for $p$ in the intersection of these sets, we define

$$
\kappa_{i}(p)=\lim _{t \downarrow 0} \frac{\left(\kappa_{i}\right)_{t}\left(p+t n_{p}\right)}{1-t\left(\kappa_{i}\right)_{t}\left(p+t n_{p}\right)},
$$

where $n_{p}$ is the outer unit normal to $\partial K$ in $p$, and $\left(\kappa_{i}\right)_{t}, i=1, \ldots, n$, are the principal curvatures of $\partial K_{t}$. If $K$ is $\lambda$-convex, then $K_{t}$ is $\left(\lambda^{-1}+t\right)^{-1}$-convex, and it follows from (4.8) that $\kappa_{i} \geqslant \lambda$.

Theorem 4.5. Let $K$ be an $H_{0}$-convex body in $\mathbb{R}^{n+1}$ for some constant $H_{0}>0$. Then

$$
\operatorname{Cap}(K) \geqslant(n-1) H_{0} \mathcal{H}^{n}(\partial K) .
$$

Equality holds in (4.9) if and only if $K$ is a round ball of radius $H_{0}^{-1}$.

Proof. We consider the function $v(p)=\Phi_{H_{0}}(r(p))$, where $r: \mathbb{R}^{n+1} \backslash \operatorname{int}(K) \rightarrow \mathbb{R}$ is the distance function to $K$ and $\Phi_{H_{0}}$ is the equilibrium potential of the Euclidean ball of radius $H_{0}^{-1}$ as defined in (2.6). The function $v$ is $C^{1,1}$ in $\mathbb{R}^{n+1} \backslash K$. Let $p \in \mathbb{R}^{n+1} \backslash K$ be a point such that the distance function $r$ is twice differentiable along the straight line minimizing the distance from $p$ to $\partial K$. Along this line we have

$$
\Delta v(p)=\Phi^{\prime \prime}(r(p))+\Phi^{\prime}(r(p)) n H_{r}(p),
$$

where $H_{r}$ is the mean curvature of the parallel hypersurface $\partial K_{r}$. Since $K_{r}$ is $\left(1 / H_{0}+\right.$ $r)^{-1}$-convex, we have at the regular points

$$
H_{r} \geqslant \frac{H_{0}}{1+H_{0} r} .
$$

Let $u$ be the equilibrium potential of $K$. Since $\Phi^{\prime} \leqslant 0$, we conclude from (4.10), (4.11) and (2.5), that

$$
\Delta v(p) \leqslant \Phi^{\prime \prime}(r(p))+\Phi^{\prime}(r(p)) \frac{n H_{0}}{1+H_{0} r(p)}=0=\Delta u(p),
$$

at every point $p$ where $r$ is $C^{2}$.

Let us check that $v \geqslant u$ in $\mathbb{R}^{n+1} \backslash K$. For every $t>0$, let $u_{t}$ be the equilibrium potential of $\left(K, K_{t}\right), \Phi_{t}$ the equilibrium potential in Euclidean space of $\left(\bar{B}_{1 / H_{0}}, B_{\left(1 / H_{0}\right)+t}\right)$, and $v_{t}:=\Phi_{t} \circ r$. Equation (4.12) implies that $\Delta\left(v_{t}-u_{t}\right) \leqslant 0$ in $K_{t} \backslash K$ in the sense of distributions. By the weak maximum principle, $v_{t} \geqslant u_{t}$ in $K_{t} \backslash K$. Since $v_{t}, u_{t}$ are increasing families converging to $v$ and $u$ respectively we conclude that $v \geqslant u$ in $\mathbb{R}^{n+1} \backslash K$. 
Let $\Gamma(p)=\left\{q \in \mathbb{R}^{n+1} \backslash K:|p-q|<C r(q)\right\}$ be a non-tangential cone at $p \in \partial K$. By [15, Thm. 1.7], $\nabla u$ has a non-tangential limit at $\mathcal{H}^{n}$-almost every point of the boundary of $K$, i.e., $\lim _{q \rightarrow p} \nabla u(q), q \in \Gamma(p)$, exists for $\mathcal{H}^{n}$-almost every $p \in \partial K$.

We pick $p \in \partial K$ so that $\nabla u$ extends to $p, \nabla v$ is defined in $p$ and $u$ extends continuously to $p$, and restrict them to an outer ball $B$ to $K$. Applying Hopf's maximum principle (see [9] and [7]) to $B$ we get, since $\Delta(v-u) \leqslant 0$ in $B, v \geqslant u$ in $B, v-u \in \mathcal{C}^{0}(\bar{B})$ and $u(p)=v(p)=1$ that

$$
|\nabla v|(p) \leqslant|\nabla u|(p) .
$$

Since formula (2.3) is valid for arbitrary convex sets [15, we get

$$
\begin{aligned}
\operatorname{Cap}(K)=\int_{\partial K}|\nabla u| d A & \geqslant \int_{\partial K}|\nabla v| d A \\
& =-\Phi^{\prime}(0) \mathcal{H}^{n}(\partial K)=(n-1) H_{0} \mathcal{H}^{n}(\partial K),
\end{aligned}
$$

which yields the desired inequality. Assume equality holds in (4.13). Then $|\nabla v|=$ $|\nabla u| \mathcal{H}^{n}$-almost everywhere on $\partial K$. So if $p \in \partial K$ is such that $|\nabla v|(p)=|\nabla u|(p)$, applying the maximum principle on an outer ball $B$ to $K$ we have that $u \equiv v$ on $B$ and then $u \equiv v$ on $\mathbb{R}^{n+1} \backslash K$.

So the level sets of $u$ and $v$, and their gradients, coincide in $\mathbb{R}^{n+1} \backslash K$. The level sets of $v$ are the parallel hypersurfaces $\partial K_{r}$, which are of class $C^{1,1}$, and the gradient of $v$ is equal to $-\Phi^{\prime}(r) \nabla r$, which never vanishes in $\mathbb{R}^{n+1} \backslash K$. Hence the level sets of $u$ are $C^{\infty}$ hypersurfaces since $u \in C^{\infty}\left(\mathbb{R}^{n+1} \backslash K\right)$ and $\nabla u=\nabla v \neq 0$.

Since equality holds in (4.13), it is also attained in (4.11) and (4.12). In particular, the principal curvatures of $\partial K_{r}$ are all equal to $\left(H_{0}^{-1}+r\right)^{-1}$, what implies that $\left\{K_{r}\right\}_{r>0}$ are concentric balls of radius $H_{0}^{-1}+r$. Hence $\partial K$ is a sphere of radius $H_{0}^{-1}$.

\section{REFERENCES}

[1] Stephanie B. Alexander, Locally convex hypersurfaces of negatively curved spaces, Proc. Amer. Math. Soc. 64 (1977), no. 2, 321-325. MR 0448262 (56 \#6571)

[2] Werner Ballmann, Mikhael Gromov, and Viktor Schroeder, Manifolds of nonpositive curvature, Progress in Mathematics, vol. 61, Birkhäuser Boston Inc., Boston, MA, 1985. MR MR823981 (87h:53050)

[3] Isaac Chavel, Riemannian geometry, second ed., Cambridge Studies in Advanced Mathematics, vol. 98, Cambridge University Press, Cambridge, 2006, A modern introduction. MR MR2229062 (2006m:53002)

[4] Jeff Cheeger and Shing Tung Yau, A lower bound for the heat kernel, Comm. Pure Appl. Math. 34 (1981), no. 4, 465-480. MR MR615626 (82i:58065)

[5] Graziano Crasta, Ilaria Fragalà, and Filippo Gazzola, On a long-standing conjecture by PólyaSzegö and related topics, Z. Angew. Math. Phys. 56 (2005), no. 5, 763-782. MR MR2184904 (2006i:31006)

[6] Björn E. J. Dahlberg, Estimates of harmonic measure, Arch. Rational Mech. Anal. 65 (1977), no. 3, 275-288. MR 0466593 (57 \#6470)

[7] Robert Dautray and Jacques-Louis Lions, Analyse mathématique et calcul numérique pour les sciences et les techniques. Tome 2, Collection du Commissariat à l'Énergie Atomique: Série Scientifique. [Collection of the Atomic Energy Commission: Science Series], Masson, Paris, 1985, With the collaboration of Michel Artola, Philippe Bénilan, Michel Bernadou, Michel Cessenat, Jean-Claude Nédélec, Jacques Planchard and Bruno Scheurer. MR 902801 (88i:00003a)

[8] Herbert Federer, Curvature measures, Trans. Amer. Math. Soc. 93 (1959), 418-491. MR 0110078 (22 \#961)

[9] David Gilbarg and Neil S. Trudinger, Elliptic partial differential equations of second order, Classics in Mathematics, Springer-Verlag, Berlin, 2001, Reprint of the 1998 edition. MR MR1814364 (2001k:35004)

[10] Alexander Grigor'yan, Isoperimetric inequalities and capacities on Riemannian manifolds, The Maz'ya anniversary collection, Vol. 1 (Rostock, 1998), Oper. Theory Adv. Appl., vol. 109, Birkhäuser, Basel, 1999, pp. 139-153. MR MR1747869 (2002a:31009) 
[11] Jacques Hadamard, Sur certaines propriétés des trajectoires en dynamique, J. Math. Pures Appl. 5 (1897), no. 3, 331-387 (French).

[12] David Hoffman and Joel Spruck, Sobolev and isoperimetric inequalities for Riemannian submanifolds, Comm. Pure Appl. Math. 27 (1974), 715-727. MR 0365424 (51 \#1676)

[13] Kanji Ichihara, Curvature, geodesics and the Brownian motion on a Riemannian manifold. I. Recurrence properties, Nagoya Math. J. 87 (1982), 101-114. MR MR676589 (84m:58166a)

[14] _ Curvature, geodesics and the Brownian motion on a Riemannian manifold. II. Explosion properties, Nagoya Math. J. 87 (1982), 115-125. MR MR676590 (84m:58166b)

[15] David Jerison, A Minkowski problem for electrostatic capacity, Acta Math. 176 (1996), no. 1, 1-47. MR MR1395668 (97e:31003)

[16] Terry Lyons and Dennis Sullivan, Function theory, random paths and covering spaces, J. Differential Geom. 19 (1984), no. 2, 299-323. MR MR755228 (86b:58130)

[17] Steen Markvorsen and Vicente Palmer, Transience and capacity of minimal submanifolds, Geom. Funct. Anal. 13 (2003), no. 4, 915-933. MR MR2006562 (2005d:58064)

[18] _ How to obtain transience from bounded radial mean curvature, Trans. Amer. Math. Soc. 357 (2005), no. 9, 3459-3479 (electronic). MR MR2146633 (2006i:58050)

[19] Peter Petersen, Riemannian geometry, second ed., Graduate Texts in Mathematics, vol. 171, Springer, New York, 2006. MR MR2243772 (2007a:53001)

[20] George Pólya and Gábor Szegö, Isoperimetric Inequalities in Mathematical Physics, Annals of Mathematics Studies, no. 27, Princeton University Press, Princeton, N. J., 1951. MR MR0043486 (13,270d)

[21] Jeffrey Rauch, An inclusion theorem for ovaloids with comparable second fundamental forms, J. Differential Geometry 9 (1974), 501-505. MR 0353225 (50 \#5709)

[22] Rolf Schneider, Convex bodies: the Brunn-Minkowski theory, Encyclopedia of Mathematics and its Applications, vol. 44, Cambridge University Press, Cambridge, 1993. MR MR1216521 (94d:52007)

[23] Gábor Szegö, Über einige Extremalaufgaben der Potentialtheorie, Math. Z. 31 (1930), no. 1, 583-593. MR MR1545137

[24] — Über einige neue Extremaleigenschaften der Kugel., Math. Z. 33 (1931), 419-425 (Ger$\operatorname{man})$.

[25] _ On the capacity of a condenser, Bull. Amer. Math. Soc. 51 (1945), 325-350. MR MR0011872 (6,227f)

[26] Martina Zähle, Integral and current representation of Federer's curvature measures, Arch. Math. (Basel) 46 (1986), no. 6, 557-567. MR 849863 (88a:53072)

Departamento de Geometría y Topología, Facultad de Ciencias, Universidad de Granada,

E-18071 Granada, EsPaña

E-mail address: ahurtado@ugr.es

Departament de Matemàtiques - Institut of New Imaging Technologies, Universitat Jaume I, E-12071 CASTEllón, España

E-mail address: palmer@mat.uji.es

Departamento de Geometría y Topología, Facultad de Ciencias, Universidad de Granada,

E-18071 Granada, España

E-mail address: ritore@ugr.es 